\title{
Zohra Sakaly, un témoignage
}

Entretien avec Monique Sicard et Claire Riffard

\section{Zohra Sakaly}

\section{(2) OpenEdition}

1 Journals

Édition électronique

URL : https://journals.openedition.org/coma/453

DOI : $10.4000 /$ coma.453

ISSN : 2275-1742

\section{Éditeur}

Institut des textes \& manuscrits modernes (ITEM)

\section{Référence électronique}

Zohra Sakaly, «Zohra Sakaly, un témoignage », Continents manuscrits [En ligne], 3 | 2014, mis en ligne le 08 novembre 2014, consulté le 12 janvier 2023. URL : http://journals.openedition.org/coma/453 ; DOI : https://doi.org/10.4000/coma.453

Ce document a été généré automatiquement le 12 janvier 2023.

\section{(c)}

Creative Commons - Attribution - Pas d'Utilisation Commerciale - Pas de Modification 4.0 International - CC BY-NC-ND 4.0

https://creativecommons.org/licenses/by-nc-nd/4.0/ 


\title{
Zohra Sakaly, un témoignage
}

\author{
Entretien avec Monique Sicard et Claire Riffard
}

\section{Zohra Sakaly}

\section{NOTE DE L'AUTEUR}

Le texte qui suit est issu de deux entretiens qui ont eu lieu, à l'invitation de Jean Loup Pivin $^{1}$, au siège de Revue noire, le 10 juillet et le 26 septembre 2013.

Zohra Sakaly est la fille du photographe sénégalo-marocain Abdourahmane Sakaly, installé au Mali de 1948 à sa mort en 1988. Il a acquis par sa pratique photographique une très grande notoriété. Abdourahmane Sakaly a vu le Mali sous colonisation française basculer en 1960 vers une indépendance dominée par la figure du président Modibo Keïta ; il a assisté ensuite à l'arrivée du régime militaire de Moussa Traoré. « À l'époque de Sakaly, dit Jean Loup Pivin [entretien du 10 juillet 2013], l'homme nouveau est au rendez-vous. L'Indépendance, c'est l'accès à la modernité en direct. Les costumes, les cravates, les postes de radio, si présents dans les photos de studio, participent de cela. » 
Fig. 1 : Zohra Sakaly

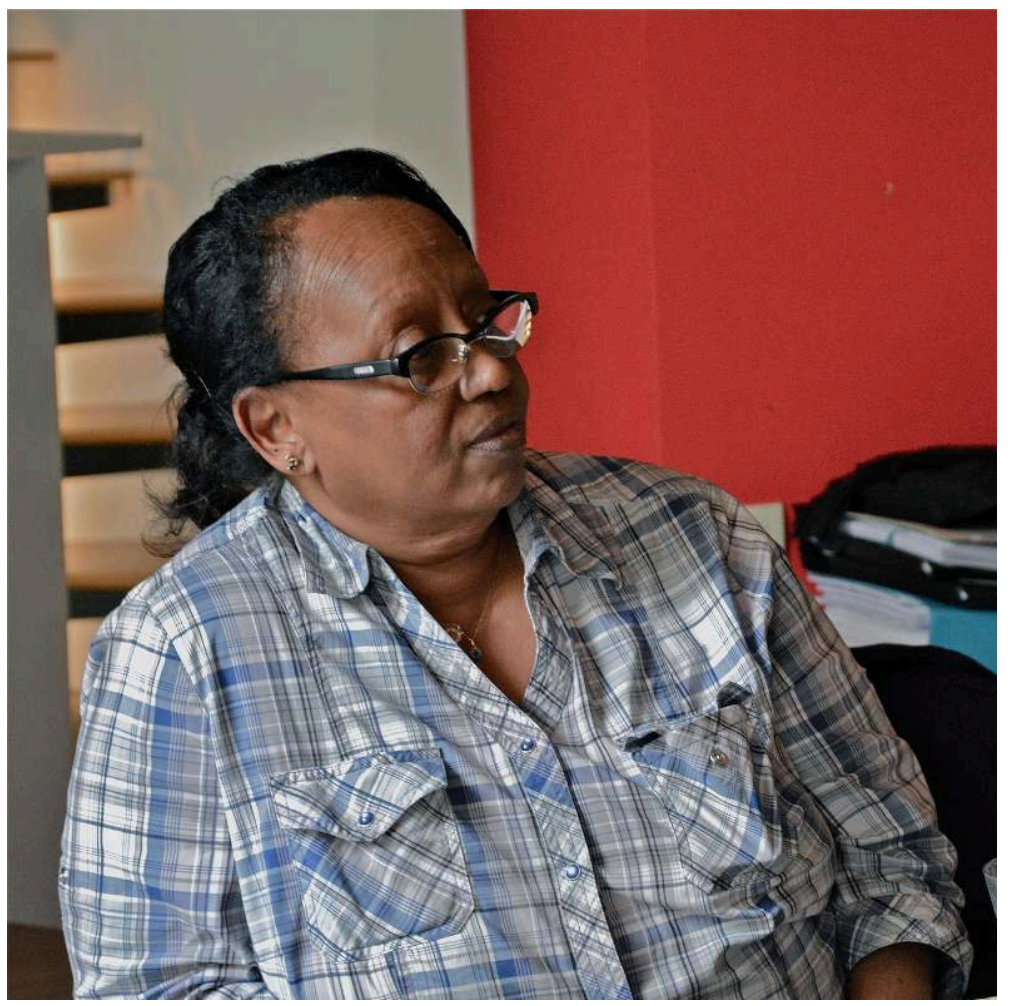

Photographie ITEM.

Le 10 juillet 2013.

Format jpeg.

Siège de la Revue noire.

(c) ITEM

\section{Premiers mots}

Mon père, Abdourahmane Sakaly, est né le 3 avril 1926 au Sénégal. Il était photographe. 
Fig. 2 : Abdourahmane Sakaly (1926-1988)

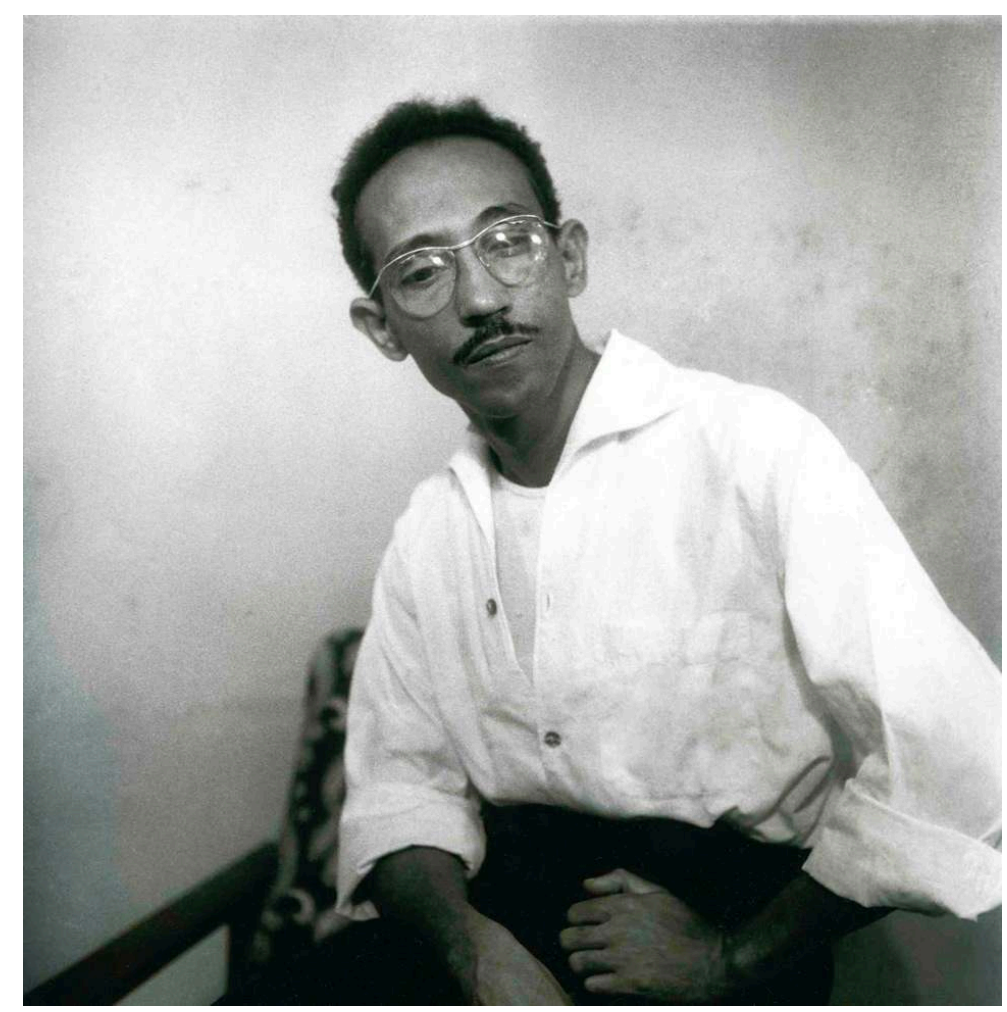

Photographe anonyme.

Fonds privé.

(c) Zohra Sakaly.

1 Il me racontait qu'au début, il développait les films dans une boîte à conserve. Mais je n'en ai pas le souvenir, car j'ai été élevée par mes grands-parents. À l'époque, les femmes laissaient souvent les grands-parents élever les enfants.

2 Mes parents sont partis à Bamako en 1948 ; moi, j'ai été élevée à Dakar. J'ai passé du temps avec mes parents pour la première fois à l'âge de 16 ans. J'appelais mon père Abou ; mes grands-parents, papa, maman. Nous étions une fratrie de sept (quatre filles et trois garçons). Une sœur est morte en bas âge et mon frère Nabi est mort il y a 6 ans. J'ai aussi deux demi-frères : Youssouf et Wahab. Mon petit frère, Sidi, vit au Maroc, le pays dont mon père est originaire ; il s'est installé à Kenitra. Mon père lui a appris la photographie.

\section{Premières photos}

3 Mon père a quitté le Sénégal quand il avait 18 ans, avec ses trois frères. Ses frères faisaient tous de la photo. L'un des frères a voulu ouvrir un studio à Dakar. Un autre est devenu cameraman au Maroc. Ils ont tous baigné dans la photo. Sakaly est d'abord allé au Congo, à Kinshasa, dans l'ancien Congo belge, puis au Mali, parce que là-bas il n'y avait pas de photographes, pas de concurrence. Il allait photographier les gens à Tombouctou, à Gao, dans la brousse, car on leur demandait de faire une photo pour la carte d'identité. 
Dans ses débuts, lorsque mon père faisait des photos en brousse, les gens avaient peur, beaucoup fuyaient quand il mettait la tête sous le voile de la chambre photographique. Ils avaient peur de celui qui était là, sous un drap noir. Cela, il me l'a raconté, car je n'étais pas encore née. Je suis née en 1951, le 2 juillet.

5 Après le studio du Mali, Sakaly a ouvert un second studio à Dakar, à proximité de la grande mosquée. Mais ce studio de Dakar n'est resté ouvert qu'une année. Lui, Sakaly, préférait le Mali. C'est le Mali qui lui a tout donné, et il a voulu être enterré au Mali.

6 Le studio du Mali a été fermé en 2007, à la mort de mon jeune frère Nabi, qui en avait repris la direction en 1988, à la mort de mon père.

\section{Photographe, mode d'emploi}

7 Sakaly avait un ami qui possédait à Bamako un magasin de matériel photo. On y parlait français, et beaucoup de produits étaient importés de France. Il avait un autre ami, Claude Rollin, qui travaillait au Centre culturel français et qui aidait beaucoup mon père à se procurer son matériel. Les pellicules étaient parfois aussi achetées en Côte d'Ivoire. On utilisait Kodak et Ilford mais Sakaly aimait bien Kodak. Un monsieur qui voyageait souvent en Côte d'Ivoire lui en rapportait.

8 A l'époque, c'était très difficile de préparer les produits (révélateur, fixateur). Pour savoir si la préparation qu'il avait effectuée était correcte, mon père goûtait le produit.

Il avait du bon matériel : deux agrandisseurs, plusieurs appareils-photo. Il a d'abord eu une chambre de prise de vue, puis un Rolleiflex et un Leica avec un pied. Au départ, il utilisait des plaques de verre pour les négatifs.

Son magasin était composé de plusieurs pièces: une entrée climatisée, avec un comptoir, des fauteuils, des boîtes pour le classement des photos, une vitrine où il exposait des photos. Il était le seul photographe à avoir une vitrine. Il avait mis un grand tirage d'un portrait de moi! Il mettait aussi un sapin à Noël, enfin, un filao qui faisait penser à un sapin! Au fond du magasin à droite, on arrivait dans une arrièrecour, avec une pièce climatisée pour les archives et une petite pièce qui servait de studio. Dans le studio, on était dans les vapeurs. Il faisait très chaud. La pièce était petite. Très souvent mon père était en marcel. Il adorait cela.

11 Pour les portraits, on disposait un rideau, qui était changé pour chacun. Le client choisissait (tout bleu, ou avec des fleurs...), mais pour les photographies d'identité, le rideau était uni. Là, c'était mon père qui choisissait. L'éclairage était réalisé par des spots, en haut et sur les côtés, qui fonctionnaient grâce à des générateurs. Les gens posaient. Il y avait même une colonne surmontée d'un pot de fleurs. Sakaly intervenait pour limiter l'appréhension des clients. Il posait les mains sur leurs épaules, il les rassurait. Les femmes mettaient leurs bijoux, leurs colliers en or, leurs grandes boucles d'oreille de Mopti... Il mettait un peu de poudre sur leur nez.

Dans un coin du studio, elles trouvaient un miroir, des crayons de maquillage. Les enfants posaient avec leurs jouets. Sakaly essayait de leur parler mais il s'exprimait mal en bambara. Il faisait des grimaces derrière l'appareil photo afin de faire sourire les enfants. Ou bien, il leur disait: "souris! je te donnerai un bonbon". C'était alors, effectivement, un bonbon, ou bien une pièce de monnaie. 
réalisait des photos pour les fêtes musulmanes ou chrétiennes. Pour les fêtes chrétiennes, pour Noël, il installait un petit sapin avec une petite lumière et des guirlandes, à côté de la personne photographiée. Les musulmans conservaient le sapin ! pour l'Aïd, il n'utilisait aucun objet particulier. Il disait, en désignant les personnes photographiées : «ils sont déjà tout décorés, avec leurs boubous, leurs bijoux... ». faut que j'allume la radio!». Mon père lui a dit : « parce que tu crois qu'on va capter le son de la radio dans la photo?»

fes chasseurs sont venus avec des lionceaux. Je les trouvais mignons, j'aurais aimé en emporter un. Mon père n'a pas voulu : « Toi, le lion, dehors ! ».

Il plaçait le travail au-dessus de tout. Il disait qu'il était là pour le boulot. Le studio était ouvert en continu tous les jours, sauf le dimanche où il ouvrait vers $18 \mathrm{~h}$. Il restait ouvert jusqu'à minuit, une heure du matin. Il y avait parfois la queue.

18

Mon père quittait la maison vers $6 \mathrm{~h}-6 \mathrm{~h} 30 \mathrm{du}$ matin. La veille, il avait réalisé les prises de vue et développé les pellicules. Vers $8 \mathrm{~h}-9 \mathrm{~h}$, les gens venaient chercher les photos d'identité prises la veille après-midi. Il faisait les tirages le matin, c'était sec très vite. Lorsque mon père n'était pas content d'un tirage, il le déchirait et le jetait. Il réalisait deux ou trois prises pour chaque photo. Ensuite, il choisissait. Lorsque son assistant, Barou, effectuait les tirages, il n'était jamais loin, il contrôlait la qualité. Toutes les photos étaient recadrées. Ce qu'appréciaient les clients, c'était la qualité des images.

\section{Zora se fait tirer le portrait}


Fig. 4 : Zohra Sakaly avec une amie

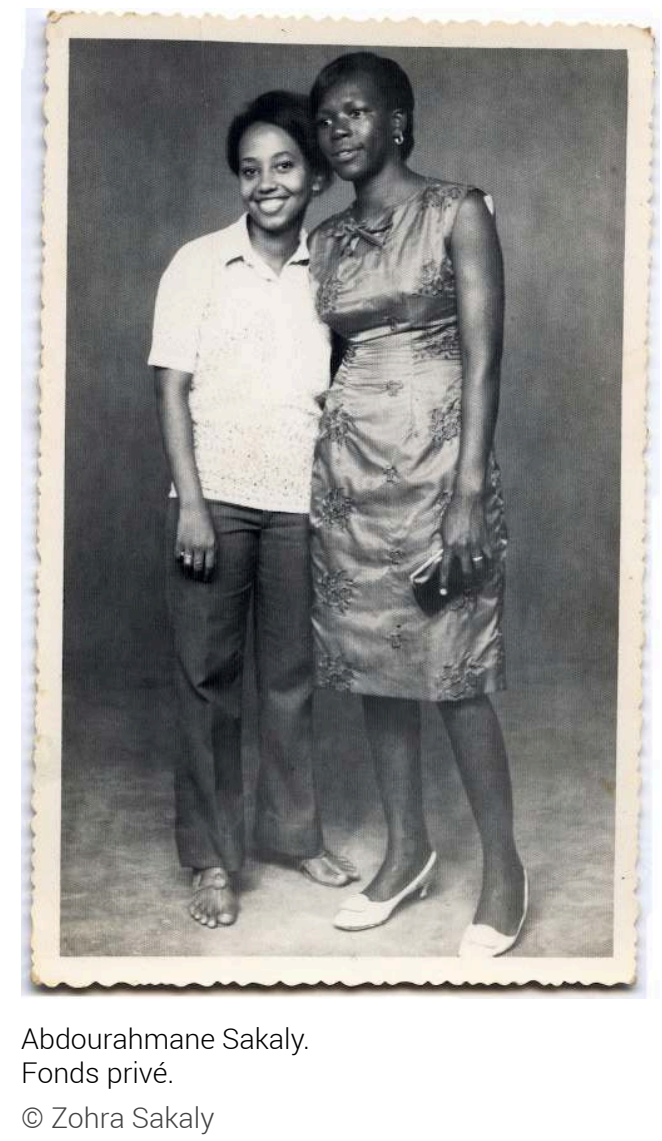

19 Dans ces années-là où j'ai rejoint mon père au Mali, j'étais ce qu'on peut appeler une fille gâtée. J'allais avec mes copines dans les magasins, dans les boîtes de nuit, je faisais envoyer la note à mon père, il payait tout. J'achetais des vêtements importés de France, chez Kouloumba. Un jour, il m'a vue arriver au studio avec un nouvel habit tout neuf, il m'a dit : "Ca te va bien, je vais te faire une photo ». Alors il m'a dit de regarder sur le côté, comme on voit sur la photo. 
Fig. 5 : Zohra Sakaly

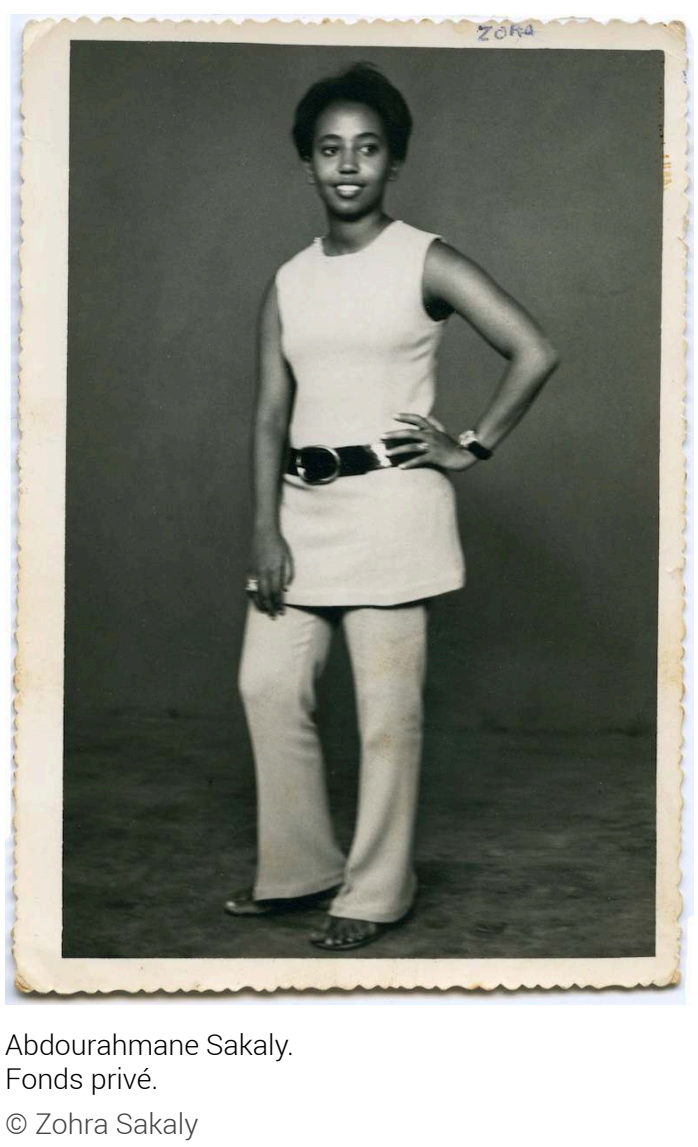

20 Cette autre photo, c'est avec mes amies. On s'amusait beaucoup ensemble, on faisait les folles. En haut à gauche, c'est Line, puis à droite mes deux amies antillaises Marie et Irène. En bas à gauche, à côté de moi, c'est Hortense. Nous sommes toujours restées amies, jusqu'à aujourd'hui. Pour la pose, il nous avait dit de nous accroupir, et j'ai eu peur de tomber tellement on riait, alors on s'est pris les mains avec Hortense, et il était content de la pose, il a dit « ne bougez plus », mais on avait très envie de rire ! 
Fig. 6 : Zohra Sakaly avec ses amies

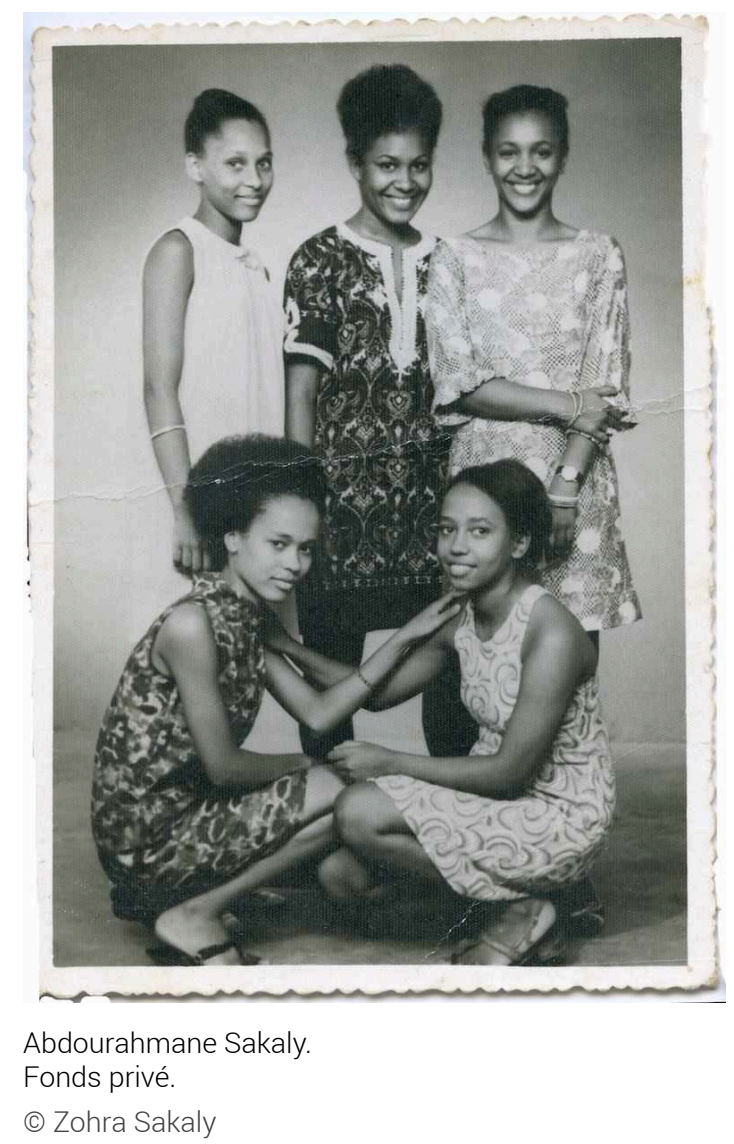

21 Mon père aussi aimait bien s'amuser. Un jour, il m’a piégée! Je sortais de la cuisine quand «clac», j'entends le déclenchement automatique de l'appareil. Il y avait une photo, mais pas de photographe!

\section{Un studio au cœur du quartier}

La question de l'étranger est au cœur. Nous étions des étrangers : mon père est d'origine marocaine. Mais les Maliens disaient : « on va avoir confiance en cet étranger qui est plus sérieux que les photographes maliens ». Ils disaient qu'il était le meilleur photographe.

Déjà, le fait de voir marqué "Studio Sakaly » au-dessus de la boutique signifiait qu'il n'était pas malien. Sakaly n'est pas un nom malien. Mais les Maliens pensaient : «si c'est un étranger, il va faire de belles choses ».

Sakaly n'a jamais eu aucun problème. Tout le monde le connaissait, même les fous et les aveugles, même les petits mendiants, à la gare de Bamako : «le studio Sakaly, c'est par là ! ". Lorsque les gens prenaient un taxi, ils demandaient au chauffeur " Amenez-moi chez Sakaly ». Cela suffisait. Puis, quand le client du taxi voulait payer la course, le chauffeur refusait. C'était Sakaly lui-même qui payait.

Lorsque je tenais la caisse du studio, il arrivait que les personnes qui venaient se faire photographier n'aient pas d'argent. On leur disait : « ce n'est pas grave, tu l'apporteras 
demain, quand tu viendras chercher la photo ». On le marquait sur le reçu. Il n'y avait pas de problème.

Sakaly avait des concurrents, mais il était réputé. Le studio était situé Medina Coura, à côté du grand marché; c'était un quartier bourgeois, où habitait aussi Amadou Hampâté Bâ. Mon père avait installé deux bancs devant la façade du studio. C'était ce qu'on appelle un grain. Les gens venaient après le travail : des ministres, des peintres, des enseignants, le patron de la police.... On offrait du thé. C'est ainsi que j'ai conduit deux ans sans permis. Le policier disait «Sakaly, fais venir ta fille, on va lui donner son permis! » mais mon père n'a jamais voulu.

$\mathrm{Au}$ Mali, les musulmans ne demandent jamais de photographie des personnes décédées. Mais les chrétiens le souhaitent parfois. Mon frère l'a fait. Il disait : « Je m'en fous, c'est une commande ». Quand Thierry Sabine et Balavoine ont eu leur accident d'avion ${ }^{2}$, la police est venue chercher Sakaly au studio. Elle n'avait pas de photographe. Mais finalement, c'est mon frère qui a réalisé le reportage. Il est parti à la morgue. Il disait que c'était terrible à voir.

Elle est venue le chercher aussi lorsqu'il y a eu un vol important dans une station service Shell. Le chef de la police allait au grain. Il ne payait pas. Mon père l'insultait : «la prochaine fois, vous n'aurez pas mes photos. Si vous ne payez pas, j'envoie ma fille ».

Il était aussi convoqué parfois au consulat du Maroc, par exemple. Lorsqu'il était appelé, il y allait.

Les photographies des anciens présidents du Mali, Modibo Keita et Moussa Traoré, sont de lui, pour la plupart. Par exemple, il avait réalisé une grande affiche du Président Keita qui a été exposée sur le mur d'un supermarché de Bamako. Modibo Keïta était présent à l'inauguration du magasin. C'était en 1967. J'étais là lorsqu'a été réalisée la première photographie officielle de Moussa Traoré, après le coup d'état de 1968. Il a fait plusieurs prises de vue. L'une dans son bureau, avec le boubou traditionnel; l'autre avec un couvre-chef militaire. Puis encore une autre avec sa femme, à l'extérieur, en utilisant deux gros flashs. On est resté là-bas longtemps : peutêtre deux heures. Il fallait prendre le temps de l'habillage. La lumière du jour a été privilégiée.

Ensuite, j'allais très souvent au Palais. Je discutais avec sa femme et son beau-père. On parlait de tout et de rien. Le Palais était sur les hauteurs mais le président préférait loger en bas. Bien que dictateur, il était très gentil.

Il faisait aussi les photos d'école, classe par classe. Dans son grain, il y avait des directeurs d'école. Ils lui disaient : « Viens photographier mon école », et il y allait.

\section{Un style}

Quand les Maliens voyaient une photo de mon père, ils reconnaissaient son style. Ils savaient que c'était lui. Ils reconnaissaient le studio, le carrelage à fleurs, le rideau, mais aussi la qualité de la photo.

Je peux vous raconter une histoire à ce sujet. Quand mon père est mort, mon frère a donné des des négatifs à Sidibe. Lui les a vendus. Mais quand j'ai visité l'exposition du Palais de la Découverte en 2012, j'y ai vu une photo de mon père. J'ai reconnu le drap de fond, le sol, alors que la photographie était présentée comme étant réalisée par Malick Sidibe. 
Mon père aimait beaucoup le noir et blanc. Il avait raison. Il disait qu'avec le noir et blanc, on voit tout, on voit plus nettement les traits du visage. Il adorait son Leica. C'était son appareil préféré. Quand la couleur est arrivée, Sakaly a trouvé que c'était « n'importe quoi ». Au début, il disait qu'il ne voulait pas toucher à cela. Mais ensuite... il a aussi photographié en couleur.

Quand mon père ratait une photo - qu'elle était floue ou qu'il y avait des taches -, il déchirait l'image tout de suite. Elle ne sortait pas du studio.

Il nous parlait beaucoup de la fameuse photo de l'hôtel de ville de Robert Doisneau. C'était pour lui un très grand photographe.

Il ne parlait pas trop de ce qu'il lisait. Il recevait des revues sur la photographie qu'il avait commandées en France grâce à la Maison de presse de Bamako.

\section{La transmission}

Sakaly a formé beaucoup de jeunes. Il était sévère. Il leur donnait des coups de pied au derrière en leur disant : " vous allez apprendre comme la vie est dure » ou bien « je vais te découper le derrière ». Vissé, qui a été apprenti chez mon père, m’a dit un jour : « Il me poussait, il me criait dessus, il m'insultait mais c'est grâce à lui que je suis devenu photographe. Je ne regrette rien. Il disait : "Je vous montre une fois, deux fois, trois fois. Si ça ne rentre pas, c'est que vous êtes des idiots". » Vissé a tenu à faire les photos du mariage de ma fille en 2004. Il lui a dit «c'est grâce à ton grand-père que j'ai appris le métier ».

Mon père n'a pas travaillé pour ses enfants. Il nous disait: «Faites comme moi : je travaille, j'en profite!». Je lui disais qu'il avait raison. Quand il est mort, j'ai dit à mon frère Nabi : « On te laisse le studio ».

Mon père a commencé à m'apprendre la photo à Dakar. Puis je l'ai rejoint au Mali en 1967. Au départ j'étais derrière la caisse. Plus tard, mon père m'a envoyée faire des reportages quand il n'avait pas le temps de s'occuper de tout.

Il m'a dit : «Oui, je vais t'apprendre à faire des photos pour les anniversaires, pour les mariages. Tu vas tout faire toi-même. Tu vas faire des photos pour le calendrier. Tu vas photographier ce que tu vois. " Car au Mali, c'est lui qui était chargé d'illustrer le calendrier d'Air Mali. C'est lui qui décidait de ses prises de vue. Après, les commanditaires choisissaient ce qu'ils voulaient - il a photographié des masques dogons, le fleuve Niger, avec les vaches, les enfants qui se baignent -.... J'ai donc fait des photos en 1967-68.

$\mathrm{Au}$ début je m'amusais, on faisait des photos de groupe avec mes copines et le déclencheur automatique, on rigolait. Mon père m'a donné des conseils pour la prise de vue : «Fais très attention. Quand tu fais la photo, au moment de déclencher, ne respire même plus. » Il me conseillait de tourner le dos au soleil et m'expliquait : "sur ton Rolleiflex, tu soulèves ce bouton, tu tournes celui-là... » Il me montrait tout, plusieurs fois, ensuite il me disait «maintenant, c'est à toi de faire, tu t'occupes de tout ! Il me disait: "voilà ce que tu dois faire quand il fait nuit." Il me donnait beaucoup, beaucoup, de conseils. Quand je quittais Bamako pour réaliser des photos, il me disait : "Marque tout ce que je te dis au stylo ». Je ne sais plus où sont les carnets sur lesquels j'ai noté tout cela. Ma sœur ne se rappelle plus non plus. 
Un jour, il m'a envoyée faire un reportage à Mopti. Là, j'ai vécu une aventure : mon appareil est tombé en panne. Il m'en a fait envoyer un second par avion. Je devais photographier des chasseurs de serpents. Et là, on m'a dit : « Viens voir les chasseurs de serpents. » Ils mettaient la main dans les trous, ça faisait peur! Les serpents et moi, on n'est pas d'accord!

Je n'ai jamais eu de problème pour prendre les gens en photo. Je disais : «je suis la fille à Sakaly, le photographe ! ». Il m'arrive, même à Paris, que des gens qui me voient dans la rue s'écrient: "C'est la fille à Sakaly! Ah oui, oui, Bamako!» Les gens me reconnaissent. C'est vrai aussi que dans son studio, il avait accroché une très grande photo de moi-même. Il l'avait réalisée avec son agrandisseur tourné face contre terre.

Quand je revenais des reportages, il choisissait les photos. Pour les tirages, il me disait: « tu as les trois bacs ». Je faisais tout, je plaçais des pinces à linge en fin de compte pour les images soient bien planes. Quand j'effectuais les tirages, mon père était à côté de moi. Je suis la seule fille à laquelle il a appris la photo; j'étais sa préférée. Mais il l'a aussi enseigné à mon jeune frère, qui est aujourd'hui au Maroc. Il fait des impressions sur Tee-shirt, ce qui reste dans la continuité.

\section{Les dernières années}

Quand il a commencé à prendre des photos en couleur, je n'étais plus là. Au début de ses prises de vue en couleur, il a voulu faire les tirages lui-même. Mais il y avait toujours quelque chose à acheter. Et puis, il fallait changer tout le matériel, l'agrandisseur... Or lui, son matériel, c'était la prunelle de ses yeux.

On lui a dit: « Tu sais, on t'installe une machine pour faire des tirages. » Il a dit : « Non, je ne veux pas. Mon studio, il a toujours fonctionné comme ça. » Finalement, il a confié ses tirages à réaliser à quelqu'un.

Entre photographes, ils se connaissaient, il n'y avait pas de problème. Lorsque l'un d'eux avait un problème avec ses appareils, il pouvait appeler Sakaly. Il faisait aussi, parfois, les développements et les tirages pour d'autres. Notamment les photographes ambulants, qui partaient en brousse, parfois à 100 ou $200 \mathrm{kms}$ de Bamako, avec leur mobylette, pour faire les photos d'identité.

Comme on l'appelait souvent pour des reportages, parfois, il en avait deux ou trois en même temps. Alors, il envoyait quelqu'un d'autre. Il pouvait sous-traiter à Malik Sidibe, à Seïdou Keïta. Il leur disait: "Tu diras que tu viens de ma part». Il y avait cette entente qui était là. «Sakaly, tu peux me dépanner ce soir ? Je n'ai pas de pellicule ».

Vers la fin de sa vie, alors qu'il s'est rendu compte qu'il n'était pas en forme, il a donné à chacun de nous un autoportrait de lui-même en format 18X24. Sur cette photographie, il regarde légèrement de côté ; il porte un beau costume. Il avait un certain nombre de beaux costumes.

31 À son enterrement, il y a eu du monde pendant une semaine. La cour était immense mais elle était pleine. Je pense que les Maliens n'auraient pas accepté qu'on rapatrie le corps au Maroc. Une rue doit porter son nom. Il y a déjà un stade, à Bamako : le stade Sakaly.

Un jour ma grand-mère, qui est décédée, m'avait montré des photos qu'il avait prises pendant mon enfance; je portais des couettes et des petits nœuds. Il y avait à l'époque des barboteuses avec des petits pois, des manches bouffantes. Ma grand-mère n'a 
jamais voulu nous donner les photos. Elles sont restées dans la maison familiale de Casablanca. Je ne sais plus où elles sont aujourd'hui.

\section{Les archives}

Sur les tirages, on imprimait un tampon avec la date et l'indication "Studio Sakaly». Les tirages n'étaient pas signés. Il n'y avait pas de planche-contact. Mon père conservait soigneusement les négatifs, il ne les donnait jamais. Il les glissait soigneusement dans des demi-enveloppes beiges, qu'il rangeait dans des boîtes jaunes et noires, des boîtes Kodak ; il en avait deux par mois, une qui allait du $1^{\mathrm{er}}$ au 15 du mois, l'autre du 16 au 31. Il expliquait aux clients qu'avec ce système ils pourraient commander un deuxième tirage de la photo. Quand il était content d'une photo, il en gardait un tirage pour lui.

Dans leur grande majorité, ces images sont à Bamako. À Paris, à Revue Noire, se trouvent environ 1000 négatifs mais là-bas, à Bamako, ce sont 400000 négatifs. Parmi les 400000 clichés, il y a beaucoup de photographies d'identité. Ce nombre pose des problèmes de transport. Il faut de lourdes malles. Je dois aller sur place, discuter avec mon frère afin de tenter de les ramener. J'aimerais rapatrier en France les clichés de Sakaly qui sont à Bamako car, là-bas, qui va les utiliser? Moi, je préfère le pays où je vis. Des Américains, des Canadiens ont demandé des négatifs de Sakaly. Mais moi, je ne vis pas au Canada!

Il y a sept ou huit ans, dans le magazine Géo, il y avait un portrait fait par Sakaly. Au Musée National de Bamako, une exposition a eu lieu, il y a deux ans. Aujourd'hui, certaines photographies sont sur internet.

\section{L'image de fin}

Ah, une dernière chose, c'est vrai qu'on n'en n'a pas parlé avant, en fait mon père était très myope ! Il voyait vraiment mal, et quand il cassait ses lunettes, il fallait en urgence en faire commander en France, il avait trouvé une astuce pour accélérer la livraison. C'était son principal outil de travail, ses lunettes! D'ailleurs, sur les photos, on le voit toujours avec ses lunettes fumées. Mon père, c'était un fameux numéro !

\section{NOTES}

2. Le 14 janvier 1986 (NDLR).

1. Jean Loup Pivin est directeur des éditions Revue noire, à Paris. 
INDEX

Mots-clés : Photographie, Afrique, Jean Loup Pivin, Revue noire, Sakaly Abdourahmane, Mali Index géographique : Afrique, Mali

\section{AUTEUR}

ZOHRA SAKALY

Fille du photographe Abdourahmane Sakaly 